\title{
Fuzzy Guaranteed Cost Control for Uncertain Singular Systems with State and Input delays
}

\author{
Rui Yang ${ }^{1}$ Tianmin Huang ${ }^{1}$ Cuihong Wang ${ }^{1}$ Li Zou $^{2}$ \\ ${ }^{1}$ School of Electrical Engineering, Southwest Jiaotong University, Chengdu 610031, P. R. China \\ ${ }^{2}$ School of Computer and Information Technology, Liaoning Normal University, Dalian 116029, P.R. China
}

\begin{abstract}
Based on T-S fuzzy model, the robust guaranteed cost control problem is studied for a class of uncertain singular systems with state and input delays. Sufficient conditions are provided for the construction of a state feedback guaranteed cost controller. These conditions are given in terms of the feasibility of linear matrix inequalities (LMIs) and guarantee that the closed-loop systems are quadratically stable. Moreover, an upper bound of the guaranteed cost is also obtained. A numerical example is provided to demonstrate the effectiveness of the proposed method.
\end{abstract}

Keywords: T-S fuzzy model, Guaranteed cost control, Linear matrix inequality (LMI), Uncertain time-delay system, Singular system

\section{Introduction}

In the past few years, there has been rapidly growing interest in fuzzy control of nonlinear systems, and there have been many successful applications. In most of these applications, the so-called Takagi-Sugeno(T-S) type fuzzy model is used to represent a nonlinear system; then based on this fuzzy model, a fuzzy controller is designed. Taniguchi $T$. et al.[1] established T-S fuzzy singular model, which can describe or approximate a wider class of complex nonlinear systems. From then on, some results on this model have been reported.

Time delays and parameter uncertainties are frequently encountered in the behavior of many physical processes and very often they are the main cause for poor performance and instability of control systems. In view of this, the robustness issue of timedelay and parameter uncertain systems is a topic of great practical importance which has attracted a great deal of interest for several decades. In recent years, some authors have paid their attention to control of nonlinear systems with time-delay and parameter uncertainties by using T-S fuzzy models. Cao and Frank first considered the T-S based fuzzy control for nonlinear systems with time delay[2, 3]. The stability analysis and synthesis of these systems via linear T-S fuzzy models was addressed in terms of LMIs.

In the control of uncertain systems, it is desirable to design a control system, which is not only stable but also guarantees an adequate level of performance. One approach to this problem is the so called guaranteed cost control approach in which a fixed quadratic Lyapunov function is used to establish an upper bound on the closed-loop value of an integral quadratic cost function. Recently, there are many results on fuzzy guaranteed cost control for normal nonlinear systems [4-8]. E. Boukas proposed a new approach to develop the results on fuzzy guaranteed cost control[4]. Bing Chen et al. investigated guaranteed cost control for TS fuzzy systems with state and input delays and also derived stability and stabilization conditions which were delay-dependent for state delay and delayindependent for input delay[5]. To the best of our knowledge, there have been few research results on fuzzy guaranteed cost control for uncertain singular nonlinear systems with state and input delays.

We present in this paper a guaranteed cost controller for uncertain singular systems with both state and input time delays based on T-S fuzzy model. Sufficient conditions for the existence of fuzzy guaranteed cost control law for the systems are obtained. A new Lyapunov functional and LMI approach are employed to analyze the stability and design the guaranteed cost controller. Finally, a simulation example is provided to demonstrate the effectiveness of the proposed method.

Notations: $R^{n}$ denotes the $n$-dimensional Euclidean space, $\|\cdot\|$ refers to either the Euclidean vector norm or the induced matrix 2-norm. $C\left([-\tau, 0], R^{n}\right)$ denotes the Banach space of continuous vector functions mapping the interval $[-\tau, 0]$ into $R^{n}$, the superscript 'T, denotes matrix transposition and $I$ is the identity matrix of appropriate dimensions, the notion $X>Y$ (respectively, $X \geq Y$ ), where $X$ and $Y$ are symmetric matrices, means that the matrix $X-Y$ is positive definite (respectively, positive semidefinite).

\section{Problem Statement}


Consider the following uncertain singular system described by T-S model with state-delay and inputdelay, the rule of the model is

Controller rule $i$ : if $\xi_{1}(t)$ is $M_{i 1}$ and $\xi_{2}(t)$ is $M_{i 2} \cdots$ and $\xi_{p}(t)$ is $M_{i p}$, then

$\left\{\begin{aligned} E \dot{x}(t)= & \left(A_{i}+\Delta A_{i}(t)\right) x(t)+\left(A_{1 i}+\Delta A_{1 i}(t)\right) x\left(t-\tau_{1}\right) \\ & +\left(B_{i}+\Delta B_{i}(t)\right) u(t)+\left(B_{1 i}+\Delta B_{1 i}(t)\right) u\left(t-\tau_{2}\right) \\ x(t)= & \varphi(t), t \in\left[-\max \left(\tau_{1}, \tau_{2}\right), 0\right] . \quad i=1,2, \cdots, k\end{aligned}\right.$ where $\xi(t)=\left[\xi_{1}(t), \xi_{2}(t), \cdots, \xi_{p}(t)\right]$ is the premise variable vector, $M_{i j}$ is the fuzzy set, $x(t) \in R^{n}$ is the state vector, $u(t) \in R^{m}$ is the control input, $E, A_{i}, A_{1 i}, B_{i}, B_{1 i}$ are known real constant matrices with appropriate dimensions and rankE $=r<n$. The scalars $\tau_{1}>0$ and $\tau_{2}>0$ are constant state and input time delays, respectively. $\Delta A_{i}, \Delta A_{1 i}, \Delta B_{i}, \Delta B_{1 i}$ are time-varying matrices representing norm-bounded parameter uncertainties and are assumed to be of the following form

$\left[\begin{array}{llll}\Delta A_{i} & \Delta A_{1 i} & \Delta B_{i} & \Delta B_{1 i}\end{array}\right]=D F(t)\left[\begin{array}{llll}G_{i} & G_{1 i} & E_{i} & E_{1 i}\end{array}\right]$ where $D, G_{i}, G_{1 i}, E_{i}$ and $E_{1 i}$ are known real constant matrices with appropriate dimensions, the uncertain real time-varying matrices $F(t)$ satisfying

$$
F(t)^{T} F(t) \leq I
$$

$\varphi(t) \in C[-\tau, 0]$ is a compatible continuous vector valued continuous initial function.

By using singleton fuzzy generator, product of fuzzy inference and weighted average defuzzifier, the global fuzzy control system can denote as the following form

$$
\left\{\begin{aligned}
E \dot{x}(t)= & \sum_{i=1}^{k} h_{i}(\xi(t))\left[\left(A_{1}+\Delta A_{1}(t)\right) x(t)+\left(A_{i i}+\Delta A_{i i}(t)\right) x\left(t-\tau_{1}\right)\right. \\
& \left.+\left(B_{1}+\Delta B_{i}(t)\right) u(t)+\left(B_{1 i}+\Delta B_{1 i}(t)\right) u\left(t-\tau_{2}\right)\right] \\
x(t)= & \varphi(t), t \in\left[-\tau_{1}, 0\right] . \quad i=1,2, \cdots, k
\end{aligned}\right.
$$

where

$$
\begin{aligned}
& h_{i}(\xi(t))=\beta_{i}(\xi(t)) / \sum_{i=1}^{k} \beta_{i}(\xi(t)) \geq 0, \\
& \beta_{i}(\xi(t))=\prod_{j=1}^{p} M_{i j}\left(\xi_{j}(t)\right) \geq 0, \\
& \sum_{i=1}^{k} h_{i}(\xi(t))=1 .
\end{aligned}
$$

$M_{i j}\left(\xi_{j}(t)\right)$ is the degree of the membership of $\xi_{j}(t)$ in $M_{i j}$
To stabilize the class of systems under consideration, we assume that the state feedback fuzzy controller is described by the following

Controller rule $i$ : if $\xi_{1}(t)$ is $M_{i 1}$ and $\xi_{2}(t)$ is $M_{i 2} \cdots$ and $\xi_{p}(t)$ is $M_{i p}$, then$$
u(t)=K_{i} x(t)
$$

The overall state feedback fuzzy controller is represented by

$$
u(t)=\sum_{j=1}^{k} h_{j}(\xi(t)) K_{j} x(t) .
$$

where $K_{j}$ are the local control gains. For simplicity, by using the following notions

$$
\begin{array}{ll}
\bar{A}_{i}=A_{i}+\Delta A_{i}(t), & \bar{A}_{1 i}=A_{1 i}+\Delta A_{1 i}(t), \\
\bar{B}_{i}=B_{i}+\Delta B_{i}(t), & \bar{B}_{1 i}=B_{1 i}+\Delta B_{1 i}(t), \\
h_{j}(t)=h_{j}(\xi(t)) . &
\end{array}
$$

and substituting the controller (2) to the system (1), we can get the closed-loop system

$$
\begin{aligned}
E \dot{x}(t) & =\sum_{i=1}^{k} h_{i}(t) \sum_{j=1}^{k} h_{j}(t)\left[\left(\bar{A}_{1}+\bar{B}_{i} K_{j}\right) x(t)+\bar{A}_{1 i} x\left(t-\tau_{1}\right)\right] \\
& +\sum_{i=1}^{k} h_{i}(t) \sum_{j=1}^{k} h_{j}\left(t-\tau_{2}\right) \bar{B}_{1 i} K_{j} x\left(t-\tau_{2}\right)
\end{aligned}
$$

Given a positive definite symmetric matrices $Q$ and $R$, the cost function associated with system (1) is

$$
J=\int_{0}^{\infty}\left[x^{T}(t) Q x(t)+u^{T}(t) R u(t)\right] d t
$$

In this paper we are interested in developing sufficient conditions for designing a state feedback controller that stabilizes the system and at the same time guarantees that the chosen cost is bounded for all admissible uncertainties. Sufficient conditions are established for this purpose to synthesize such controller by the LMI method.

Before we give the main result of this paper, some definitions and lemmas are introduced which will be used in the rest of the paper.

Definition 1 The nominal fuzzy singular system (1) with $u(t)=0$ is regular, if there exists $s \in C$, such that

$$
\operatorname{det}\left[s E-\sum_{i=1}^{k} h_{i}(\xi(t))\left(A_{i}+\Delta A_{i}\right)\right] \neq 0, \forall t \geq 0 \text {. }
$$

Definition 2 The nominal fuzzy singular system (1) with $u(t)=0$ is impulse-free, if

$$
\operatorname{deg} \operatorname{det}\left[s E-\sum_{i=1}^{k} h_{i}(\xi(t))\left(A_{i}+\Delta A_{i}\right)\right]=\operatorname{rank} E
$$

Definition 3 The regular and impulse-free fuzzy singular system is quadratically stable if $\dot{V}(t)<0$ 
with $V(x(t))=x^{T}(t) E^{T} P x(t)$, where nonsingular matrix $P$ satisfying $E^{T} P=P^{T} E$.

Lemma 1[9] Given matrices $A, D, E$ and $R>0$ with appropriate dimensions. Let $F(t)$ be of appropriate dimensions and satisfying $F^{T}(t) F(t) \leq I$, then the following inequality hold.

(a) For any scalar $\varepsilon>0$,

$$
D F(t) E+E^{T} F^{T}(t) D^{T} \leq \varepsilon D D^{T}+\varepsilon^{-1} E^{T} E
$$

(b) If for some scalar $\varepsilon>0$, such that $\varepsilon I-E R E^{T}>0$, then

$$
\begin{aligned}
& (A+D F(t) E) R(A+D F(t) E)^{T} \\
& \leq A R\left(R-\varepsilon^{-1} R E^{T} E R\right)^{-1} R A^{T}+\varepsilon D D^{T}
\end{aligned}
$$

Lemma 2[10] For any constant matrix $M>0$ and scalar $\sigma>0$, vector function $x(t):[0, \sigma] \rightarrow R^{n}$ such that the integral concerned are well defined, the following inequality holds

$$
\left[\int_{t-\sigma}^{t} x(s) d s\right]^{T} M\left[\int_{t-\sigma}^{t} x(s) d s\right] \leq \sigma \int_{t-\sigma}^{t} x^{T}(s) M x(s) d s
$$

\section{Main Results}

In this section, an existence condition of guaranteed cost controller for system (1) is proposed, which not only guarantees the closed-loop system is asymptotically stable for all admissible uncertainties, but also provides an upper bound for the cost function.

Theorem 1 Consider the singular system (1) and the cost function (4), then the corresponding closedloop system (3) is asymptotically stable if there exist matrices $S>0, L>0$ and nonsingular matrix $P$, such that the following matrix inequalities hold

$$
\begin{aligned}
& E^{T} P=P^{T} E \geq 0 \\
& {\left[\begin{array}{cc}
\Theta(i, j) & P^{T} \bar{A}_{1 i} \\
\bar{A}_{1 i}^{T} P & -S
\end{array}\right]<0}
\end{aligned}
$$

where

$$
\begin{aligned}
\Theta(i, j)= & P^{T}\left(\bar{A}_{i}+\bar{B}_{i} K_{j}\right)+\left(\bar{A}_{i}+\bar{B}_{i} K_{j}\right)^{T} P+P^{T} \bar{B}_{1 i} L^{-1} \bar{B}_{1 i}^{T} P \\
& +S+K_{i}^{T} L K_{i}+Q+\left(\frac{K_{i}+K_{j}}{2}\right)^{T} R\left(\frac{K_{i}+K_{j}}{2}\right)
\end{aligned}
$$

then the controller (2) is a guaranteed cost controller for system (3) in terms of the cost function (4) and an upper bound of the guaranteed cost is

$$
\begin{aligned}
J^{*}= & \varphi^{T}(0) E^{T} P \varphi(0)+\int_{-\tau_{1}}^{0} \varphi^{T}(\alpha) S \varphi(\alpha) d \alpha \\
& +\sum_{i=1}^{k} \int_{-\tau_{2}}^{0} h_{i}(\alpha) \varphi^{T}(\alpha) K_{i}^{T} L K_{i} \varphi(\alpha) d \alpha .
\end{aligned}
$$

Proof. Define Lyapunov function

$$
V=V_{1}+V_{2}+V_{3}
$$

$$
\begin{aligned}
& V_{1}=x^{T}(t) E^{T} P x(t) \\
& V_{2}=\int_{t-\tau_{1}}^{t} x^{T}(\alpha) S x(\alpha) d \alpha \\
& V_{3}=\sum_{i=1}^{k} \int_{t-\tau_{2}}^{t} h_{i}(\alpha) x^{T}(\alpha) T_{i} x(\alpha) d \alpha
\end{aligned}
$$

It is easy from (3) and (5) to see that the time derivative of $V_{1}$ along any trajectory of the closed-loop system (3) is given by

$$
\begin{aligned}
\dot{V}_{1}= & 2 x^{T}(t) P^{T} E \dot{x}(t) \\
= & \sum_{i=1}^{k} \sum_{j=1}^{k} h_{i}(t) h_{j}(t) 2 x(t)^{T} P^{T}\left[\left(\bar{A}+\bar{B}_{i} K_{j}\right) x(t)+\bar{A}_{1 i} x\left(t-\tau_{1}\right)\right] \\
& +\sum_{i=1}^{k} h_{i}(t) \sum_{j=1}^{k} h_{j}\left(t-\tau_{2}\right) 2 x^{T}(t) P^{T} \bar{B}_{1 i} K_{j} x\left(t-\tau_{2}\right)
\end{aligned}
$$

Since $L>0$, we can get the following inequality

$$
\begin{aligned}
& 2 x^{T}(t) P^{T} \bar{B}_{1 i} K_{j} x\left(t-\tau_{2}\right) \\
& \leq x^{T}(t) P^{T} \bar{B}_{1 i} L^{-1} \bar{B}_{1 i}^{T} P x(t)+x^{T}\left(t-\tau_{2}\right) K_{j}^{T} L K_{j} x\left(t-\tau_{2}\right)
\end{aligned}
$$

Then substituting (10) into (9) yields

$$
\begin{aligned}
\dot{V}_{1} & \leq \sum_{i=1}^{k} \sum_{j=1}^{k} h_{i}(t) h_{j}(t) x^{T}(t)\left[P^{T}\left(\bar{A}+\bar{B}_{i} K_{j}\right)+\left(\bar{A}+\bar{B}_{i} K_{j}\right)^{T} P\right. \\
& \left.+P^{T} \bar{B}_{1 i} L^{-1} \bar{B}_{1 i}^{T} P\right] x(t)+\sum_{i=1}^{k} \sum_{j=1}^{k} h_{i}(t) h_{j}(t) 2 x^{T}(t) P^{T} \bar{A}_{1 i} x\left(t-\tau_{1}\right) \\
& +\sum_{i=1}^{k} h_{i}\left(t-\tau_{2}\right) x^{T}\left(t-\tau_{2}\right)\left(K_{i}^{T} L K_{i}-T_{i}\right) x\left(t-\tau_{2}\right)
\end{aligned}
$$

The derivative of $V_{2}$ and $V_{3}$ in (8) are obtained, respectively

$$
\begin{aligned}
\dot{V}_{2} & =x^{T}(t) S x(t)-x^{T}\left(t-\tau_{1}\right) S x\left(t-\tau_{1}\right) \\
\dot{V}_{3} & =\sum_{i=1}^{k} h_{i}(t) x^{T}(t) T_{i} x(t) \\
& -\sum_{i=1}^{k} h_{i}\left(t-\tau_{2}\right) x^{T}\left(t-\tau_{2}\right) T_{i} x\left(t-\tau_{2}\right)
\end{aligned}
$$

Then we have

$$
\begin{aligned}
& \dot{V}=\sum_{i=1}^{k} \sum_{j=1}^{k} h_{i}(t) h_{j}(t)\left[\begin{array}{c}
x^{T}(t) \\
x^{T}\left(t-\tau_{1}\right)
\end{array}\right] \\
& {\left[\begin{array}{cc}
\left(\begin{array}{c}
P^{T}\left(\bar{A}+\bar{B}_{1} K_{j}\right)+\left(\bar{A}+\bar{B}_{1} K_{j}\right) P \\
+P^{T} \bar{B}_{i i} L^{-1} \bar{B}_{1 i}^{T} P+S+T_{i}
\end{array}\right) P^{T} \bar{A}_{i j} \\
\bar{A}_{1 i}^{T} P & -S
\end{array}\right]\left[\begin{array}{ll}
x(t) & \left.x\left(t-\tau_{1}\right)\right] \\
+\sum_{i=1}^{k} h_{i}\left(t-\tau_{2}\right) x^{T}\left(t-\tau_{2}\right)\left(K_{i}^{T} L K_{i}-T_{i}\right) x\left(t-\tau_{2}\right)
\end{array}\right.}
\end{aligned}
$$


On the other hand, from (2), $u(t)$ can be rewritten

$$
u(t)=\sum_{j=1}^{k} h_{j}(t) K_{j} x(t)=\sum_{i=1}^{k} \sum_{j=1}^{k} h_{i}(t) h_{j}(t) \frac{K_{i}+K_{j}}{2} x(t)
$$

thus,

$$
\begin{aligned}
u^{T}(t) R u(t)= & \sum_{i=1}^{k} \sum_{j=1}^{k} h_{i}(t) h_{j}(t)\left(\frac{K_{i}+K_{j}}{2} x(t)\right)^{T} \\
& \cdot R \sum_{i=1}^{k} \sum_{j=1}^{k} h_{i}(t) h_{j}(t)\left(\frac{K_{i}+K_{j}}{2} x(t)\right) \\
\leq & \sum_{i=1}^{k} \sum_{j=1}^{k} h_{i}(t) h_{j}(t)\left(\frac{K_{i}+K_{j}}{2} x(t)\right)^{T} \\
& \cdot R\left(\frac{K_{i}+K_{j}}{2} x(t)\right)
\end{aligned}
$$

Therefore

$$
\begin{aligned}
& x^{T}(t) Q x(t)+u^{T}(t) R u(t) \\
& \leq \sum_{i=1}^{k} \sum_{j=1}^{k} h_{i}(t) h_{j}(t)\left[\begin{array}{c}
x^{T}(t) \\
x^{T}\left(t-\tau_{1}\right)
\end{array}\right] \\
& {\left[\begin{array}{cc}
Q+\left(\frac{K_{i}+K_{j}}{2}\right)^{T} R \frac{K_{i}+K_{j}}{2} & 0 \\
0 & 0
\end{array}\right]\left[\begin{array}{ll}
x(t) & \left.x\left(t-\tau_{1}\right)\right] .
\end{array}\right.}
\end{aligned}
$$

Choose $T_{i}=K_{i}^{T} L K_{i}$, so it follows from (6), (11) and (12) that

$$
\begin{aligned}
& \dot{V} \leq \sum_{i=1}^{k} \sum_{j=1}^{k} h_{i}(t) h_{j}(t)\left[\begin{array}{c}
x^{T}(t) \\
x^{T}\left(t-\tau_{1}\right)
\end{array}\right]\left[\begin{array}{cc}
\Theta(i, j) & P^{T} \bar{A}_{1 i} \\
\bar{A}_{1 i}^{T} P & -S
\end{array}\right] \\
& \cdot\left[x(t) \quad x\left(t-\tau_{1}\right)\right]-x^{T}(t) Q x(t)-u^{T}(t) R u(t) \\
& <-x^{T}(t) Q x(t)-u^{T}(t) R u(t)<0
\end{aligned}
$$

So the closed-loop system (3) is quadratically stable.

Furthermore, integrating both sides of the above inequality from 0 to $\infty$ produces

$$
\begin{aligned}
& J=\int_{0}^{\infty}\left[x^{T}(t) S x(t)+u^{T}(t) R u(t)\right] d t \\
& \leq \varphi(0)^{T} E^{T} P \varphi(0)+\int_{-\tau_{1}}^{0} \varphi^{T}(\alpha) Q \varphi(\alpha) d \alpha \\
& \quad+\sum_{i=1}^{k} \int_{-\tau_{2}}^{0} h_{i}(\alpha) \varphi^{T}(\alpha) K_{i}^{T} L K_{i} \varphi(\alpha) d \alpha=J^{*}
\end{aligned}
$$

This completes the proof.

In order to apply the LMI box in Matlab software, we need to transform (5) and (6) into the following LMIs.

Theorem 2 Consider the singular system (1) and the cost function (4), if there exist matrices $Z>0, N>0$, nonsingular matrix $X$, matrices $F_{i}(i=1, \cdots, k)$ and positive real numbers $\beta_{i}$ and $\varepsilon_{i j}$ satisfying

$$
\begin{aligned}
& E X=X^{T} E^{T} \geq 0 \\
& {\left[\begin{array}{cc}
-\beta_{i} I & G_{1 i} \\
G_{1 i}^{T} & -L
\end{array}\right]<0}
\end{aligned}
$$

$$
\left[\begin{array}{cccccc}
\Omega A_{\mathrm{i} i} X & X^{T} G_{i}+F_{j}^{T} E_{i}^{T} & F_{i}^{T} & B_{i} N E_{i i}^{T} & X^{T} & \left(\frac{F_{i}+F_{j}}{2}\right)^{T} \\
-Z & X^{T} G_{i}^{T} & 0 & 0 & 0 & 0 \\
& -\varepsilon_{i j} I & 0 & 0 & 0 & 0 \\
& & -N & 0 & 0 & 0 \\
& & & -\beta_{i} I+E_{i i} N E_{1 i}^{T} & 0 & 0 \\
& & & & -Q^{-1} & 0 \\
& & & & 0 & -R^{1}
\end{array}\right]<0
$$

where

$$
\begin{aligned}
\Omega= & A_{i} X+X^{T} A_{i}^{T}+B_{i} F_{j}+F_{j}^{T} B_{i}^{T} \\
& +B_{1 i} N B_{1 i}^{T}+\left(\varepsilon_{i j}+\beta_{i}\right) D D^{T}+Z
\end{aligned}
$$

then the controller (2) is a guaranteed cost controller for system (3) in terms of the cost function (4) and the gains of the state feedback controller are given by $K_{i}=F_{i} X^{-1}, i=1, \cdots, k$. Moreover, an upper bound of the guaranteed cost is

$$
\begin{aligned}
J^{*}= & \varphi^{T}(0) E^{T} X^{-1} \varphi(0)+\int_{-\tau_{1}}^{0} \varphi^{T}(\alpha) X^{-T} Z X^{-1} \varphi(\alpha) d \alpha \\
& +\sum_{i=1}^{k} \int_{-\tau_{2}}^{0} h_{i}(\alpha) \varphi^{T}(\alpha) X^{-T} F_{i}^{T} N^{-1} F_{i} X^{-1} \varphi(\alpha) d \alpha .
\end{aligned}
$$

Proof. The inequality (6) in theorem 1 can be written as

$$
\begin{aligned}
& {\left[\begin{array}{cc}
\Xi(i, j) & P^{T} A_{1 i} \\
A_{1 i}^{T} P & -S
\end{array}\right]+\left[\begin{array}{cc}
\Delta \Xi(i, j) & P^{T} \Delta A_{1 i} \\
\Delta A_{1 i}^{T} P & 0
\end{array}\right]} \\
& +\left[\begin{array}{cc}
P^{T} \bar{B}_{1 i} L^{-1} \bar{B}_{1 i}^{T} P & 0 \\
0 & 0
\end{array}\right]<0
\end{aligned}
$$

where

$$
\begin{aligned}
\Xi(i,, j)= & P^{T}\left(A_{i}+B_{i} K_{j}\right)+\left(A_{i}+B_{i} K_{j}\right)^{T} P+S \\
& +K_{i}^{T} L K_{i}+Q+\left(\frac{K_{i}+K_{j}}{2}\right)^{T} R\left(\frac{K_{i}+K_{j}}{2}\right) \\
\Delta \Xi(i,, j)= & P^{T}\left(\Delta A_{i}+\Delta B_{i} K_{j}\right)+\left(\Delta A_{i}+\Delta B_{i} K_{j}\right)^{T} P
\end{aligned}
$$

i.e.

$$
\begin{aligned}
& {\left[\begin{array}{cc}
\Xi(i, j) & P^{T} A_{1 i} \\
A_{1 i}^{T} P & -S
\end{array}\right]+\left[\begin{array}{c}
P^{T} \\
0
\end{array}\right] D F\left[G_{i}+E_{i} K_{j} \quad G_{1 i}\right]} \\
& +\left(\left[\begin{array}{c}
P^{T} \\
0
\end{array}\right] D F\left[G_{i}+E_{i} K_{j} \quad G_{1 i}\right]\right)^{T}+\left[\begin{array}{cc}
P^{T} \bar{B}_{1 i} L^{-1} \bar{B}_{1 i}^{T} P & 0 \\
0 & 0
\end{array}\right]<0
\end{aligned}
$$

Applying Schur complement, the inequality (14) is equivalent to 


$$
\beta_{i} I-G_{1 i} L^{-1} G_{1 i}^{T}>0
$$

By the term (a) and (b) in lemma 1 and (16), it is sufficient to prove that for some scalar $\varepsilon_{i j}>0$, $\beta_{i}>0$ and matrix $L>0$ such that

$$
\begin{aligned}
& {\left[\begin{array}{cc}
\Xi(i, j) & P^{T} A_{1 i} \\
A_{1 i}^{T} P & -S
\end{array}\right]+\varepsilon_{i j}\left[\begin{array}{cc}
P^{T} D D^{T} P & 0 \\
0 & 0
\end{array}\right]} \\
& +\varepsilon_{i j}^{-1}\left[\begin{array}{cc}
\left(G_{i}+E_{i} K_{j}\right)^{T}\left(G_{i}+E_{i} K_{j}\right) & \left(G_{i}+E_{i} K_{j}\right)^{T} G_{1 i} \\
G_{1 i}^{T}\left(G_{i}+E_{i} K_{j}\right) & G_{1 i}^{T} G_{1 i}
\end{array}\right] \\
& +\left[\begin{array}{ccc}
P^{T} B_{1 i} L^{-1}\left(L^{-1}-\beta_{i}^{-1} L^{-1} E_{1 i}^{T} E_{1 i} L^{-1}\right)^{-1} L^{-1} B_{1 i}^{T} P+\beta_{i} P^{T} D D^{T} P & 0 \\
0 & 0
\end{array}\right] \\
& <0
\end{aligned}
$$

Due to the relation

$$
\begin{aligned}
& \left(L^{-1}-\beta_{i}^{-1} L^{-1} E_{1 i}^{T} E_{1 i} L^{-1}\right)^{-1} \\
& =L-E_{1 i}^{T}\left(\beta_{i} I-E_{1 i} L^{-1} E_{1 i}^{T}\right)^{-1} E_{1 i},
\end{aligned}
$$

then

$$
\begin{aligned}
& \quad P^{T} B_{1 i} L^{-1}\left(L^{-1}-\beta_{i}^{-1} L^{-1} E_{1 i}^{T} E_{1 i} L^{-1}\right)^{-1} L^{-1} B_{1 i}^{T} P \\
& =P^{T} B_{1 i} L^{-1} B_{1 i}^{T} P \\
& \quad-\quad P^{T} B_{1 i} L^{-1} E_{1 i}^{T}\left(\beta_{i} I-E_{1 i} L^{-1} E_{1 i}^{T}\right)^{-1} E_{1 i} L^{-1} B_{1 i}^{T} P
\end{aligned}
$$

So substituting (18) into (17) yields an inequality and applying Schur complement repeatedly to the inequality, it is straightforward to verify that this yields

$$
\left[\begin{array}{cccccc}
\Phi P^{T} A_{i} & \left(G+E_{i} K_{j}\right)^{T} & K_{i}^{T} & P^{T} B_{i} L^{-1} E_{i i}^{T} & I & \left(\frac{K_{I}+K_{j}}{2}\right)^{T} \\
-S & G_{i}^{T} & 0 & 0 & 0 & 0 \\
& -\varepsilon_{i j} I & 0 & 0 & 0 & 0 \\
& & -L^{-1} & 0 & 0 & 0 \\
& & & -\beta_{i} I+E_{i j} L^{-1} E_{i i}^{T} & 0 & 0 \\
& & & & -Q^{1} & 0 \\
& & & & 0 & -R^{1}
\end{array}\right]<0
$$

where

$$
\begin{aligned}
\Phi= & P^{T}\left(A_{i}+B_{i} K_{j}\right)+\left(A_{i}+B_{i} K_{j}\right)^{T} P+S \\
& +\left(\varepsilon_{i j}+\beta_{i}\right) P^{T} D D^{T} P+P^{T} B_{1 i} L^{-1} B_{1 i}^{T} P
\end{aligned}
$$

Pre-multiply the inequality (19) by the matrix $\operatorname{diag}\left(P^{-T}, P^{-T}, I, I, I, I, I\right)$ and post-multiply by the matrix $\operatorname{diag}\left(P^{-1}, P^{-1}, I, I, I, I, I\right)$, introduce new variables $X=P^{-1}, F_{j}=K_{j} P^{-1}, Z=P^{-T} S P^{-1}$ and $N=L^{-1}$, consequently, (19) is equivalent to (15). Correspondingly, (13) becomes (5).

According to theorem 1 , the result follows immediately by replacing $P, S$ and $L$ into (7).
Remark Since (13) is an improper linear matrix inequality, we should transform it to a proper one. Without loss generality, provided that $E=\left[\begin{array}{cc}I_{r} & 0 \\ 0 & 0\end{array}\right]$, the nonsingular matrix decomposes to block matrix $X=\left[\begin{array}{ll}X_{1} & X_{2} \\ X_{3} & X_{4}\end{array}\right]$, then (13) implies $X=\left[\begin{array}{cc}X_{1} & 0 \\ X_{3} & X_{4}\end{array}\right]$, where $X_{1}$ is a symmetric positive-definite matrix, $X_{4}$ is nonsingular.

\section{Numerical Example}

In this section, we give an example to demonstrate the effectiveness of the proposed method.

Consider a uncertain singular time-delay system (1) and (2) based on T-S model with parameters as follows:

$$
\begin{aligned}
& E=\left[\begin{array}{ll}
1 & 0 \\
0 & 0
\end{array}\right], A_{1}=\left[\begin{array}{cc}
1 & 1 \\
0 & -1
\end{array}\right], A_{2}=\left[\begin{array}{ll}
0 & 1 \\
1 & 0
\end{array}\right], \\
& A_{11}=\left[\begin{array}{cc}
0 & 0 \\
0.1 & 0.2
\end{array}\right], A_{12}=\left[\begin{array}{cc}
0 & 0 \\
0.1 & 0.1
\end{array}\right], \\
& B_{1}=\left[\begin{array}{l}
0 \\
1
\end{array}\right], B_{2}=\left[\begin{array}{l}
2 \\
1
\end{array}\right], B_{11}=\left[\begin{array}{c}
0 \\
0.1
\end{array}\right], B_{12}=\left[\begin{array}{c}
0.2 \\
0
\end{array}\right], \\
& G_{1}=\left[\begin{array}{ll}
1 & 0 \\
0 & 0.1
\end{array}\right], G_{2}=\left[\begin{array}{cc}
1 & 0 \\
1 & 0.2
\end{array}\right], \quad E_{1}=\left[\begin{array}{c}
0.1 \\
0
\end{array}\right], \\
& E_{2}=\left[\begin{array}{c}
0 \\
0.2
\end{array}\right], \quad G_{11}=\left[\begin{array}{cc}
1 & 0.1 \\
0 & 1
\end{array}\right], \quad G_{12}=\left[\begin{array}{c}
0 \\
0.1
\end{array}\right], \\
& E_{11}=\left[\begin{array}{l}
0 \\
1
\end{array}\right], \quad E_{12}=\left[\begin{array}{cc}
0.1 \\
0.2
\end{array}\right], \quad D=\left[\begin{array}{cc}
0.11 & 0 \\
0 & 0.1
\end{array}\right], \\
& Q=I, \quad R=I, \quad \tau_{1}=1, \quad \tau_{2}=2, \\
& \varphi(t)=\left[\begin{array}{ll}
e^{t+1} & 0
\end{array}\right], t \in[-2,0], \\
& F=\operatorname{diag}\{r(t), s(t), q(t)\}, \text { where }|r(t) s(t) q(t)| \leq 1, \\
& h_{1}\left(x_{1}(t)\right)=1-1 / 1+e^{-x_{1}}, h_{2}\left(x_{1}(t)\right)=1 / 1+e^{-x_{1}} .
\end{aligned}
$$

Using Matlab LMI Control Toolbox to solve the LMI (15), we obtain the following feasible solution:

$$
\begin{aligned}
& X=\left[\begin{array}{cc}
0.3410 & 0 \\
-0.1695 & 0.1082
\end{array}\right], Z=\left[\begin{array}{cc}
0.6454 & 0.1714 \\
0.1714 & 0.1968
\end{array}\right], \\
& N=1.2592, \quad \beta_{1}=1.3475, \quad \beta_{2}=1.0395, \\
& \varepsilon_{11}=1.1037, \quad \varepsilon_{22}=1.0128, \\
& F_{1}=\left[\begin{array}{ll}
-0.0770 & -0.2830
\end{array}\right], F_{2}=\left[\begin{array}{ll}
-0.3298 & -0.3176
\end{array}\right] .
\end{aligned}
$$

the gains of the state feedback controller are given $\mathrm{K}_{1}=\left[\begin{array}{ll}1.2083 & -1.5490\end{array}\right], K_{2}=\left[\begin{array}{ll}-2.4265 & -2.9365\end{array}\right]$. 
An upper bound of the guaranteed cost is

$$
J^{*}=31.1745 \text {. }
$$

\section{Conclusions}

In this paper, based on the T-S fuzzy model, we have studied the fuzzy control design problem for nonlinear uncertain singular systems with state delay and input delay. An LMI-based condition for the existence of fuzzy guaranteed cost controllers has been derived. The resulting fuzzy controllers can guarantee that the closed-loop fuzzy system is quadratically stable and results in an upper bound of the closed-loop value of cost function. A numerical example is given to demonstrate the effectiveness of the proposed method.

\section{References}

[1] T. Taniguchi, K. Tanaka, K. Yamafuji and H.O. Wang, Fuzzy descriptor systems: Stability analysis and design via LMIs. Proc. of American Control of Conference, San Diego, pp. 18271831, 1999.

[2] Y.Y. Cao, P.M. Frank, Analysis and synthesis of nonlinear time-delay systems via fuzzy control approach. IEEE Trans. Fuzzy System, 8: 200-211, 2000.

[3] Y.Y. Cao, P.M. Frank, Stability analysis and synthesis of nonlinear time-delay systems via linear Takagi-Sugeno fuzzy models. Fuzzy Sets and Systems, 124: 213-229, 2001.

[4] E. Boukas, Fuzzy guaranteed cost control for nonlinear systems. Fuzzy Information Processing Society, 279-283, 2006.

[5] B. Chen, X.P. Liu, S.C. Tong, C. Lin, Guaranteed cost control for T-S fuzzy systems with state and input delays. Fuzzy Sets and Systems, 1-17, 2007.

[6] B. Chen, X.P. Liu, Fuzzy guaranteed cost control for nonlinear systems with time-varying delay. IEEE Trans. Fuzzy Systems, 13: 238-249, 2005.

[7] G.F. Ma, S. Zhong, L.K. Zhu, Y.Q. Liu, Robust analysis of fuzzy guaranteed cost control for a class of time-delay systems with uncertain parameters. Proceedings of the Third International Conference on Machine Learning and Cybernetics, Shanghai, pp. 26-29, 2004.

[8] X.P. Guan, C.L. Chen, delay-dependent guaranteed cost control for T-S fuzzy systems with time delays. IEEE Trans. Fuzzy Systems, 12: 236-249, 2004.

[9] Y. Wang, L. Xie, C.E. De Souza, Robust control of a class of uncertain nonlinear systems. Systems Control Lett. 19: 139-149, 1992.
[10] J.H. Park, O.M. Kwon, Guaranteed cost control of time-delay chaotic systems. Chaos, Solitons and Fractals, 27: 1011-1018, 2006. 